\title{
A MAXIMUM ENTROPY BASED HIERARCHICAL MODEL FOR AUTOMATIC PROSODIC BOUNDARY LABELING IN MANDARIN
}

\author{
Fangzhou Liu, Huibin Jia, Jianhua Tao \\ National Laboratory of Pattern Recognition, \\ Institute of Automation, Chinese Academy of Sciences, Beijing
}

\begin{abstract}
Modeling prosodic rhythm is of great importance for both speech synthesis and speech understanding, and it requires a large enough corpus with precise prosodic boundary labels. This paper proposes a maximum entropy (ME) based hierarchical model, which utilizes both text and acoustic features, to automatically label Mandarin prosodic boundaries. Results of comparative experiments show that, for the task of prosodic boundary detection, ME model obviously outperforms classification and regression tree (CART), and the bottom-up hierarchical framework is also significantly superior to the flat single-level framework.
\end{abstract}

Index Terms-prosodic word, prosodic phrase, intonational phrase, maximum entropy

\section{INTRODUCTION}

Prosodic structure plays an important role in both naturalness and intelligibility of speech. It breaks utterances into prosodic units which can be easily understood by people. Therefore, prosodic structure modeling is important for both speech synthesis and speech understanding. But training the model requires a very large corpus with precise prosodic boundary labels. However, manually labeling prosodic boundary for large corpus is laborious and timeconsuming. In addition, it is also not easy to keep the consistence among different labelers and even the same labeler in different time. With these limitations of manual labeling, automatic prosodic boundary labeling attracts more and more attentions now.

Various data-driven approaches, such as hidden Markov model (HMM) [1], classification and regression tree (CART) [2], CART-based hierarchical model [3][4], have been investigated to predict prosodic boundary. Based on grammatical information (such as part-of-speech, base phrase, etc) [1][3] or acoustic information (such as pitch, duration, etc) [2] or both [4], all of them achieve state-ofthe-art performance. But these methods still have their own shortcomings. HMM can only use a few discrete features like part-of-speech (POS), and is based on two strict assumptions, the Markov assumption and the output independence assumption, which have a limited validity.
Because of the well-known data fragmentation problem, CART can hardly use the highly lexicalized features like specific lexicon words, which can provide important cues for prosodic boundary detection.

Maximum entropy (ME) model has been widely used in natural language processing (NLP) [5] in recent years, since it allows the inclusion of diverse forms of information without causing data fragmentation and necessarily assuming independence between the predictors. In this paper, ME model is used to determine the probability of a lexicon word (LW) boundary being a prosodic boundary based on both text and acoustic information, and a bottomup hierarchical framework is built to find the optimal prosodic parse with the highest probability.

The rest of the paper is organized as follows. Section 2 introduces our rhythm labeled corpus and the three-tier prosodic structure adopted in the corpus. Section 3 describes the ME based hierarchical model. In section 4, text and acoustic features related to prosodic boundary are extracted from the corpus. Section 5 presents several comparative experiments and discusses the results. Final conclusions are drawn in section 6 .

\section{EXPERIMENTAL CORPUS}

\subsection{Prosodic hierarchy}

In our work, a three-tier prosodic hierarchy, which consists of prosodic word (PW), prosodic phrase (PP) and intonational phrase (IP), is defined under the sentence level. Prosodic word is the lowest constituent in the prosodic hierarchy. The middle tier is the prosodic phrase, which has a perceptive minor break at the end. Intonational phrase is the top tier with a major break at the end. The concepts of phrase accent and boundary tone in English are not very suitable for the definition of prosodic phrase and intonational phrase in Mandarin since Mandarin is a tonal language. The degree of break becomes the main cue for identifying them in real speech. Figure 1 shows an example sentence “只好求别人替他介绍一份轻松的工作 ( He has to ask others to introduce an easy job for him)", which has been decomposed into a prosodic hierarchy. 


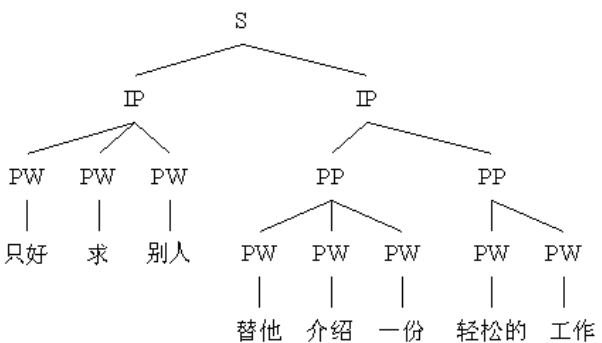

Figure 1: The prosodic hierarchy of the sentence “只好 求别人替他介绍一份轻松的工作 (He has to ask others to introduce an easy job for him) ".

\subsection{Corpus labeling}

Since there is no public Mandarin speech database available, we designed and collected a large phonetically and prosodically enriched Mandarin speech corpus. The corpus contains 6,084 sentences and around 110,000 syllables, which were uttered by a professional female speaker. By both listening to the utterances and reading the text transcriptions, four levels of break indices (B0-B3) were annotated manually by two experienced annotators, among which B0 (default, not marked explicitly) represents a nonboundary site, and B1, B2 and B3 correspond to PW, PP and IP boundary respectively. To check the consistency of annotation between the two annotators, an exploratory experiment was carried out. The two annotators were first trained on the same 100 sentences. At this stage, they were required to discuss criteria for annotation so that they could achieve agreement on most of the annotations in the 100 sentences. Then, they were asked to annotate a small subset of the corpus, which included 1,000 sentences. $96.1 \%$ of prosodic indices were agreed on by them. That is to say pretty good consistency existed between the two annotators.

\section{ME-BASED HIERARCHICAL MODEL}

\subsection{Maximum entropy model}

Maximum entropy model is a statistical classification model, which seeks the probability distribution with the maximum entropy, over the set of the ones subject to certain constraints. Such constraints force the model to match its feature expectations with those observed in the training set. A feature of maximum entropy model is defined as a nonnegative real function $f_{i}(x, y)$, in which $\mathrm{x}$ denotes the input information, and $\mathrm{y}$ denotes the category label. Given an event $(\mathrm{x}, \mathrm{y})$, the conditional probability $\mathrm{p}(\mathrm{y} \mid \mathrm{x})$ is given by:

$$
p(y \mid x)=\frac{1}{Z(x)} \prod_{i=1}^{k} \alpha_{i}^{f_{i}(x, y)}
$$

where $\alpha_{i}$ is the weight of feature $f_{i}$, which can be estimated by GIS or IIS [5] algorithms, and $\mathrm{Z}(\mathrm{x})$ is the normalization factor:

$$
Z(x)=\sum_{y} \prod_{i=1}^{k} \alpha_{i}^{f_{i}(x, y)}
$$

For more information about maximum entropy model, please refer to [5].

\subsection{Bottom-up hierarchical framework}

Since prosodic constituents have hierarchical relationships, that is to say, an IP boundary necessarily coincides with a PP boundary, and a PP boundary is inevitably a PW boundary, a bottom-up hierarchical framework is proposed. Firstly, PW boundaries are detected from all LW boundaries. Secondly, PP boundaries are detected only from those LW boundaries that have been judged as PW boundaries. Finally, IP boundaries are picked up only from the predicted PP boundaries. Figure 2 shows the flowchart of the hierarchical framework. Three ME models are trained separately to make boundary or non-boundary decisions for PW, PP and IP, respectively.

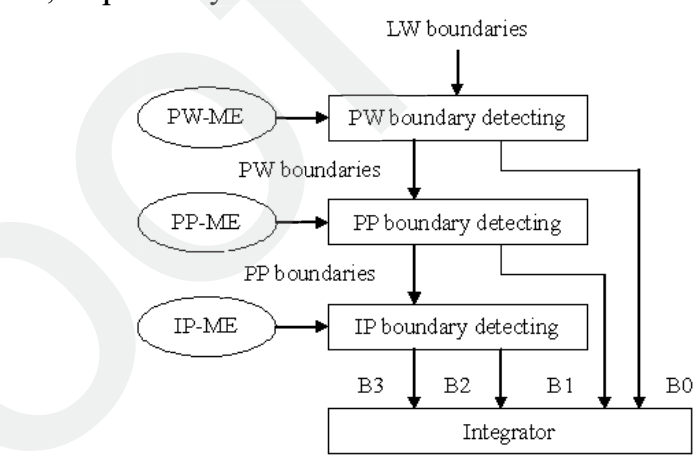

Figure 2: Flowchart of the bottom-up hierarchical framework for detecting prosodic boundaries.

\section{FEATURE SELECTION}

\subsection{Text features}

Due to the lack of sophisticated syntactic parser, only the shallow grammatical information which could be acquired easily and reliably is considered in this work. Word segmentation, POS tagging are automatically carried out by the front end module of our Mandarin TTS system, which utilizes a lexicon containing about 130,000 entries and a POS tag set consists of 39 POS tags. An accuracy of $96 \%$ for word segmentation and $91 \%$ for POS tagging were achieved. Since many special words often provide important cues for prosodic boundary location, specific lexicon words should be taken into consideration. POS is the most commonly used feature in prosodic structure prediction. The length in characters of a lexicon word is very important for predicting prosodic word boundaries, because of the disyllabic pattern of bottom rhythmic unit in Mandarin. In view of the constraints of speech production, distance from the current word boundary to the beginning of the carrying sentence is useful in detecting intonational phrase 
boundaries. All the text feature templates used in $\mathrm{ME}$ model, including the boundary types they aim at, are lists in Table 2, in which $\mathrm{W}$ denotes the lexicon word, $\mathrm{P}$ denotes POS, L denotes the length in characters of the lexicon word, DisCh denotes the distance in characters from the beginning of the sentence, and the subscript number indicates the offset. For instance, "W0P1" means the current word and POS of the immediately succeeding word. Note that the punctuation information has not been used here, as a piece of punctuation almost always implied a prosodic break, and thus can be mapped to a prosodic phrase or intonational phrase boundary through simple rules. Our work only focuses on detecting prosodic boundaries from nonpunctuation boundaries.

Table 2. Text feature templates.

\begin{tabular}{|c|c|}
\hline Symbol & Boundary type \\
\hline $\mathrm{W}_{-2}, \mathrm{~W}_{-1}, \mathrm{~W}_{0}, \mathrm{~W}_{1}, \mathrm{~W}_{2}$ & $\mathrm{~B} 1 \sim \mathrm{B} 3$ \\
\hline $\mathrm{W}_{-2} \mathrm{~W}_{-1}, \mathrm{~W}_{-1} \mathrm{~W}_{0}, \mathrm{~W}_{0} \mathrm{~W}_{1}, \mathrm{~W}_{1} \mathrm{~W}_{2}$ & $\mathrm{~B} 1 \sim \mathrm{B} 3$ \\
\hline $\mathrm{P}_{-2}, \mathrm{P}_{-1}, \mathrm{P}_{0}, \mathrm{P}_{1}, \mathrm{P}_{2}$ & $\mathrm{~B} 1 \sim \mathrm{B} 3$ \\
\hline $\mathrm{P}_{-2} \mathrm{P}_{-1}, \mathrm{P}_{-1} \mathrm{P}_{0}, \mathrm{P}_{0} \mathrm{P}_{1}, \mathrm{P}_{1} \mathrm{P}_{2}$ & $\mathrm{~B} 1 \sim \mathrm{B} 3$ \\
\hline $\mathrm{P}_{-2} \mathrm{P}_{-1} \mathrm{P}_{0}, \mathrm{P}_{-1} \mathrm{P}_{0} \mathrm{P}_{1}, \mathrm{P}_{0} \mathrm{P}_{1} \mathrm{P}_{2}$ & $\mathrm{~B} 2 \sim \mathrm{B} 3$ \\
\hline $\mathrm{W}_{0} \mathrm{P}_{1}, \mathrm{P}_{0} \mathrm{~W}_{1}$ & $\mathrm{~B} 1 \sim \mathrm{B} 3$ \\
\hline $\mathrm{L}_{-1}, \mathrm{~L}_{0}, \mathrm{~L}_{1}$ & $\mathrm{~B} 1$ \\
\hline $\mathrm{L}_{-1} \mathrm{~L}_{0}, \mathrm{~L}_{0} \mathrm{~L}_{1}$ & $\mathrm{~B} 1$ \\
\hline $\mathrm{P}_{-1} \mathrm{~L}_{-1}, \mathrm{P}_{0} \mathrm{~L}_{0}, \mathrm{P}_{1} \mathrm{~L}_{1}$ & $\mathrm{~B} 1$ \\
\hline $\mathrm{P}_{-1} \mathrm{~L}_{-1} \mathrm{P}_{0} \mathrm{~L}_{0}, \mathrm{P}_{0} \mathrm{~L}_{0} \mathrm{P}_{1} \mathrm{~L}_{1}$ & $\mathrm{~B} 1$ \\
\hline DisCh & $\mathrm{B} 3$ \\
\hline
\end{tabular}

\subsection{Acoustic features}

Although there is a general consensus that the primary acoustic cues for prosodic boundary perception are pitch reset, pre-boundary lengthening and silence, it is not the case as far as the specific acoustic correlates of each prosodic constituent. The acoustic analysis of Mandarin rhythm by Qian [6] shows that, pitch resets are observed across intonational phrase boundaries, yet, not across prosodic phrase boundaries, and prosodic word does not have noticeable marks in speech. However, according to Yang [7], both prosodic phrase and intonational phrase boundaries have significant pitch resets, and prosodic word boundaries are also signaled by a slight pitch reset and preboundary lengthening. Energy does not seem as important as pitch, duration and pause for prosodic boundary detection, but it is, after all, one of the basic prosodic features, so we still use it as a candidate feature. Since Mandarin is a tonal language, both the pitch and the duration of a syllable are closely related with its tone. The duration and the energy integral of a syllable also have a close connection with its phonetic alphabet (Pinyin being used for Mandarin). Thus, these interrelated information should be combined to form composite features. Table 3 lists all the acoustic feature templates and their target boundary types, in which Sil, Dur, F0, and Eng denote silence, duration, pitch and energy respectively, Py and Tn denote pinyin and tone, Prev and Next denote the preceding and following syllable, and DisMs is the distance in milliseconds from the beginning of the sentence. For example, "F0Delta\&TnPrev\&TnNext" indicates the difference between $\mathrm{F} 0$ of the beginning of the following syllable and the ending of the preceding syllable combined with their tones. To reduce the impact of inter-speaker and intra-speaker variation, all the acoustic features are normalized to non-negative value.

Table 3. Acoustic feature templates.

\begin{tabular}{|c|c|}
\hline Symbol & Boundary type \\
\hline Sil & B2 B3 \\
\hline DurPrev\&PyPrev & B2 B3 \\
\hline DurDelta\&PyPrev\&PyNext & B2 B3 \\
\hline F0Prev\&TnPrev & B2 B3 \\
\hline F0PrevMax\&TnPrev & B2 B3 \\
\hline F0PrevMin\&TnPrev & B2 B3 \\
\hline F0PrevMean\&TnPrev & B2 B3 \\
\hline F0Next\&TnNext & B2 B3 \\
\hline F0NextMax\&TnNext & B2 B3 \\
\hline F0NextMin\&TnNext & B2 B3 \\
\hline F0NextMean\&TnNext & B2 B3 \\
\hline F0Delta\&TnPrev\&TnNext & B2 B3 \\
\hline F0MaxDelta\&TnPrev\&TnNext & B2 B3 \\
\hline F0MinDelta\&TnPrev\&TnNext & B2 B3 \\
\hline F0MeanDelta\&TnPrev\&TnNext & B2 B3 \\
\hline EngPrev\&PyPrev & B3 \\
\hline EngNext\&PyNext & B3 \\
\hline EngDelta\&PyPrev\&PyNext & B3 \\
\hline DisMs & B3 \\
\hline
\end{tabular}

\section{EVALUATION AND DISCUSSION}

To evaluate our approach, the whole corpus is divided into the training, development and test sets according to an $8: 1: 1$ ratio. In training process, a simple count cutoff is applied to throw out features that occur very sparsely in the training set, since they generally lead to unreliable statistic results. The feature set, cutoff valve and iteration time are tested and adjusted on the development set. The evaluation criteria are precision (Pre), recall (Rec) and F-score using a $\beta$-value of 1 . Table 4 lists the precision and recall of each individual ME model with different feature sets. For PW boundary detection, only using text features gets the best results. Adding acoustic features will deteriorate slightly both the precision and recall, which is in line with Qian's argument [6] that PW does not have obvious acoustic correlates, and also challenges Yang's view [7] mentioned in section 4.2. The best performance of PP and IP boundary detection are achieved by combining both text and acoustic features. In contrast to the conclusion drawn by Qian [6], removing F0 related features will cause a significant performance decline, which also supports Yang's argument [7]. When excluding energy related features, the performance of IP ME model declines obviously, showing energy also provides useful information for IP boundary detection.

The integrated results of ME based hierarchical model are listed in table 5, which are compared with the flat framework and CART-based approach. A considerable 
performance drop is observed when the outputs from the three ME model are integrated, which may be caused by the reason that errors from the lower level model are promulgated into the higher level model. Non-hierarchical framework in which four levels of break indices are classified at the same time in a single model is obviously inferior to hierarchical framework in both the precision and recall of all three prosodic boundaries. The main two reasons are: (a) the characteristics of different prosodic constituents are not identical, for instance, PP and IP have noticeable acoustic signs, but PW does not, so it is inappropriate to predict different rhythmic units with the same features in a single model; (b) As shown in table 4, low level boundaries can be detected with pretty high accuracy, and thus they can provide valuable constraints for high level boundary detection. Under the same flat framework, CART performs significantly worse than ME, which may be due to its well-known data fragmentation problem. Highly lexicalized features like specific lexicon words even can hardly be selected as branch nodes of CART tree, while they are high-weight features in ME model.

Table 4. Performance of each individual ME model with different feature sets.

\begin{tabular}{|c|c|c|c|c|}
\hline \multirow{2}{*}{ Feature set } & $\begin{array}{c}\text { Evaluation } \\
\text { criteria }\end{array}$ & B1 & B2 & B3 \\
\hline \multirow{2}{*}{ Only text features } & Pre (\%) & $\mathbf{9 3 . 6 3}$ & 75.81 & 70.45 \\
\cline { 2 - 5 } & $\operatorname{Rec}(\%)$ & $\mathbf{9 7 . 5 1}$ & 74.51 & 51.23 \\
\hline $\begin{array}{c}\text { Only acoustic } \\
\text { features }\end{array}$ & $\operatorname{Pre}(\%)$ & 85.40 & 72.94 & 82.22 \\
\cline { 2 - 5 } & $\operatorname{Rec}(\%)$ & 88.38 & 66.18 & 45.17 \\
\hline $\begin{array}{c}\text { Text + acoustic } \\
\text { features }\end{array}$ & $\operatorname{Pre}(\%)$ & 93.01 & $\mathbf{8 3 . 1 6}$ & $\mathbf{8 8 . 8 0}$ \\
\cline { 2 - 5 } & $\operatorname{Rec}(\%)$ & 97.40 & $\mathbf{7 8 . 5 9}$ & $\mathbf{6 6 . 0 9}$ \\
\hline $\begin{array}{c}\text { PP - F0 related } \\
\text { features }\end{array}$ & $\operatorname{Pre}(\%)$ & - & 78.64 & - \\
\cline { 2 - 5 } & $\operatorname{Rec}(\%)$ & - & 71.77 & - \\
\hline $\begin{array}{c}\text { IP }- \text { Energy } \\
\text { related features }\end{array}$ & $\operatorname{Pre}(\%)$ & - & - & 87.26 \\
\cline { 2 - 5 } & $\operatorname{Rec}(\%)$ & - & - & 61.93 \\
\hline
\end{tabular}

Table 5. Performance of different approaches.

\begin{tabular}{|c|c|c|c|c|}
\hline Approach & $\begin{array}{c}\text { Evaluation } \\
\text { criteria }\end{array}$ & B1 & B2 & B3 \\
\hline \multirow{2}{*}{$\begin{array}{c}\text { ME-based } \\
\text { hierarchical } \\
\text { model }\end{array}$} & Pre (\%) & 72.21 & 67.57 & 89.04 \\
\cline { 2 - 5 } & Rec (\%) & 81.45 & 68.20 & 63.92 \\
\cline { 2 - 5 } & F-score (\%) & $\mathbf{7 6 . 5 5}$ & $\mathbf{6 7 . 8 8}$ & $\mathbf{7 4 . 4 2}$ \\
\hline $\begin{array}{c}\text { ME-based } \\
\text { single-level } \\
\text { model }\end{array}$ & Pre (\%) & 72.76 & 66.13 & 79.14 \\
\cline { 2 - 5 } & $\operatorname{Rec}(\%)$ & 78.07 & 66.43 & 67.64 \\
\cline { 2 - 5 } & F-score (\%) & 75.32 & 66.28 & 72.94 \\
\hline $\begin{array}{c}\text { CART-based } \\
\text { single-level } \\
\text { model }\end{array}$ & Pre (\%) & 65.45 & 62.06 & 77.91 \\
\cline { 2 - 5 } & Rec (\%) & 70.30 & 65.95 & 62.73 \\
\cline { 2 - 5 } & F-score (\%) & 67.79 & 63.95 & 69.50 \\
\hline
\end{tabular}

Because of differences in language, corpus, and the definition of prosodic hierarchy, comparing results obtained in different studies is not easy. The F-score of PP (67.88\%) and IP boundary $(74.42 \%)$ achieved in our study is not as high as that reported by Chou (72.44\% and $80.00 \%)$ [4]. However, considering that the ratio of break samples $(31.72 \%)$ in our corpus is higher than that of their corpus
(26.34\%), and that we do not make use of the punctuation information, the performance of our approach is satisfactory.

\section{CONCLUSION}

In this paper, a ME-based hierarchical model, which utilizes both text and acoustic features, is proposed for automatic prosodic boundary labeling in Mandarin. Compared with the flat non-hierarchical framework and CART-based approach, our method achieves the most outstanding performance. Results of comparative experiments on feature selection show that, acoustic features appear to have no effect on prosodic word prediction, pitch reset does play a role in predicting prosodic phrase, and energy also provides useful information for intonational phrase boundary detection.

Our work is based on a single-speaker corpus with the reading style for speech synthesis. Although most of the features have been normalized, we are still not sure if it will work for different speakers. So the next step is to adapt the model to spontaneous speech and multi-speaker.

\section{ACKNOWLEDGEMENTS}

This work was supported by the National Natural Science Foundation of China (No.60575032, No.70611120555) and 863 Programs (No.2006AA01Z138, No.2006AA01Z194).

\section{REFERENCES}

[1] Taylor, P. and Black, A. W., "Assigning Phrase Breaks from Part-of-Speech Sequences", Computer Speech and Language, Vol. 12, 1998, pp. 99-117.

[2] Wightman, C. W. and Ostendorf, M., "Automatic Labeling of Prosodic Patterns", IEEE Trans. on Speech and Audio Processing, Vol. 2, No. 4, 1994, pp. 469-481.

[3] Chu, M. and Qian, Y., "Locating Boundaries for Prosodic Constituents in Unrestricted Mandarin Texts", Computational Linguistics and Chinese Language Processing, February 2001, Vol.6, No.1: 61-82.

[4] Chou F. C., Tseng, C.Y. and Lee, L.S., "Automatic Segmental and Prosodic Labeling of Mandarin Speech Database", Proc. of the Fifth International Conference on Spoken Language Processing, 1998, Sydney.

[5] Adam L. Berger, Stephen A. Della Pietra, Vincent J. Della Pietra, "A Maximum Entropy Approach to Natural Language Processing", Computational Linguistics, 1996, 22(1): 39-71.

[6] Qian, Y., Chu, M., Pan, W. Y., "The Acoustic Cues of Boundaries of Prosodic Hierarchy in Mandarin", Proc. of 5th National Conference on Phonetics, 2001, pp. 70-74 (in Chinese)

[7] Yang Y., Wang B., "Acoustic Correlates of Hierarchical Prosodic Boundary in Mandarin", Proc. of Speech Prosody 2002, Aix-en-Provence (France), April 2002. 